\title{
JOURNAL.RU
}

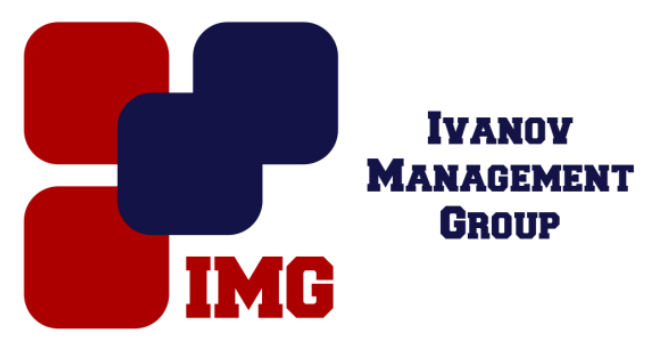

Бесланеев И.А., Курданова Мд. Х., Батырбекова Л.М., Курданова М.Х., Курданов Х.А. Центр медико-экологических исследований - филиал Федерального государственного бюджетного учреждения науки - Государственного научного иентра Российской Федераиии - Института медико-биологических проблем Российской академии наук Нальчик, Россия

doi: 10.18411/lj-30-06-2017-05

idsp 000001:1j-30-06-2017-05

\section{Дисфункция эндотелия и тиреоидный статус у больных артериальной гипертонией, жителей высокогорья}

\section{Аннотация}

В статье представлены данные о влиянии стабильных метаболитов оксида азота (NO) - нитритов и нитратов и тиреоидных гормонов (ТГ) на показатели центральной гемодинамики у здоровых лиц и больных артериальной гипертонией, жителей высокогорья. Всем пациентам проведено клиническое, инструментальное и биохимическое обследование.

Проведен сравнительный и регрессионный анализ между метаболитами оксида азота, тиреоидными гормонами и показателями центральной гемодинамики.

Показано, что у больных артериальной гипертонией при снижении концентрации стабильных метаболитов NO в крови, снижается концентрация ТГ, и изменяются параметры гемодинамики.

Ключевые слова: оксид азота, тиреоидные гормоны, артериальная гипертония, высокогорье

\section{Abstract}

In article data about influence stable metabolites nitric oxide (NO) - nitrites and nitrates and thyroids hormones (TG) on indicators of central hemodynamic in healthy faces and patients with arterial hypertension, inhabitants of high mountains is presented. To all patients clinical tool and biochemical inspection ware spent. 
It is spent comparative and regression the analysis between metabolites nitric oxide, thyroids hormones and indicators of central hemodynamic.

It is show in patients with arterial hypertension was decrease in concentration stable metabolites NO in blood, concentration $\mathrm{TG}$ decreases and hemodynamic parameters change.

Keywords: nitric oxide, thyroids hormones, arterial hypertension, high mountains

Введение. Тиреоидные гормоны (ТГ) оказывают существенное влияние на многие фундаментальные процессы и системы организма человека. ТГ влияют на метаболизм белков, липидов, углеводов, микроэлементов, рост, развитие и дифференцировку клеток, активность субклеточных и ядерных структур [1]. ТГ влияют на функции вегетативной (BНC) и центральной нервной системы (ЦНС), сердечно-сосудистой, дыхательной, иммунной, пищеварительной, гормональной и многих других систем. ТГ учувствуют в терморегуляции, скорости поглощения тканями кислорода, синтезе инсулина и оксида азота (NO), увеличивают образование эритропоэтина, гемоглобина и многих других биологически активных веществ [2,3].

Изменение концентрации NO и ТГ, приводящие к нарушениям регуляции ВНС и ЦНС, изменениям вазодилатации, клеточного состава крови являются важными факторами становления, течения АГ и развития ее осложнений $[4,5]$.

Известно, что в условиях умеренной гипоксии продукция NO увеличивается за счет адаптационных процессов: активации NO-синтаз и нитритредуктазной активности гемсодержащих протеинов и ферментов: (гемоглобина, миоглобина, ферритина, цитохромоксидазы, коэнзимов и др.), а так же эритроцитоза, снижения элиминации NO свободными радикалами и других адаптационных процессов [6,7].

Одной из важных реакций адаптации к гипоксии у жителей высокогорья является развитие гипотиреоза. Снижение секреции тиреотропного гормона (ТТГ), ТГ и йод - концентрирующей функции сопровождается снижением основного обмена и потребления кислорода тканями. При этом гемодинамические и нейрогормональные сдвиги характерные для гипотиреоза у них незначительно выражены.

Цель работы: изучить взаимосвязи между концентрацией тиреоидных гормонов, метаболитов оксида азота в крови и параметров центральной 
гемодинамики у здоровых лиц и больных артериальной гипертонией, проживающих в высокогорье.

\section{Материалы и методы}

В высокогорных районах Приэльбрусья (2100 - 3600 м. над уровнем моря) обследованы 185 пациентов: 115 больных АГ разной степени и 70 здоровых лиц:

1. 55 больных АГ 1 степени ( 24 мужчин и 31 женщин), средний возраст

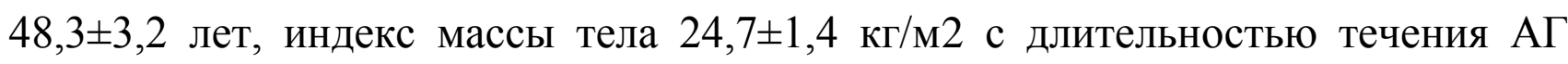
$(6,4 \pm 1,2)$ года.

2. 60 больных АГ 2 степени (27 мужчин и 33 женщин), средний возраст $54,7 \pm 2,4$ года, индекс массы тела $25,7 \pm 1,8$ кг/м2 с длительностью течения АГ $(11,7 \pm 1,3)$ года.

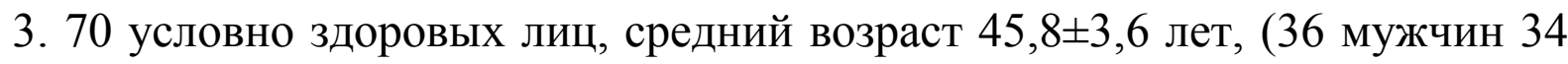
женщин), индекс массы тела 23,4 1,6 кг/м2.

Все обследованные пациенты подробно ознакомлены с методиками исследований, получено письменное информированное согласие для дальнейшего проведения исследований. Диагноз АГ верифицировали на основании данных анамнестических, клинических, биохимических и инструментальных методов исследований. Стратификацию факторов риска, общего риска, определяли в соответствие с рекомендациями, изложенными в докладе Рабочей группы по лечению артериальной гипертонии Европейского общества гипертонии и Европейского общества кардиологов (ESH/ ESC) 2013г [8].

Основными критериями включения в исследование было длительное наличие высоких цифр АД (более 140/90 мм.рт.ст.) АГ в анамнезе и факторы риска. Критерии исключения: больные АГ с уровнем АДС > 180 и АДД > 110 мм.рт.ст.; лица с признаками острых и/или обострения хронических воспалительных заболеваний; выявленной стенокардии и других форм ИБС в анамнезе; больные с нарушениями ритма и проводимости; с симптоматической артериальной гипертензией; признаками хронической сердечной недостаточности; с ассоциированными клиническими состояниями в анамнезе; больные АГ 3 степени, у которых отмена гипотензивной терапии связана с риском развития осложнений; больные принимающие лекарственную терапию влияющую на метаболизм тиреоидных гормонов и концентрацию нитритов и нитратов в крови. 


\section{Методы исследований}

Лабораторно-инструментальные и биохимические методы включали: исследование состояния сердечно-сосудистой системы $\mathrm{c}$ помощью электрокардиографии «Nihon Cohden FQW210-3-140», (Япония), эхокардиографии «Aloka SSD 500», (Япония) с количественной и качественной оценкой функции левого желудочка.

Систолическое АД (сАД) и диастолическое АД (ДАД), мм. рт. ст., определялось осциллометрическим методом с помощью автоматических тонометров для измерения артериального давления «AND» и «Omron», (Япония) и суточными многофункциональными портативными носимыми мониторами ЭКГ и АД - МЭКГ - ПН - МС «ДМС»-«СОЮЗ», «ДМС - Передовые технологии», (Россия). Среднее динамическое АД (АДСр, мм.рт.ст.) и пульсовое АД (АДП), мм.рт.ст., рассчитывали по общепринятым формулам.

продукцию оксида азота (NO) оценивали по концентрации его стабильных метаболитов: нитрит - аниона (NO2-) и нитрат - аниона (NO3-) в плазме крови и трижды отмытых эритроцитах. Концентрацию NO2- определяли в безбелковых фильтратах плазмы крови и эритроцитов с помощью реактива Грисса по методу Грина. величину экстинкции измеряли на спектрофотометре СФ-6-А (Россия) при длине волны $\lambda=543$ нм. Концентрацию NO3- определяли прямым методом с помощью спектрофотометрического метода. К 1 мл безбелковой пробы добавляли 2,5 мл бруцинового реактива. Величину экстинкции определяли при длине волны $\lambda=405$ нм [9].

Концентрацию тиреотропного гормона (ТТГ), свободного и общего трийодтиронона (с. Т3, об Т3), свободного и общего тетрайодтиронина тироксина (с. Т4, об. Т4), антитела к тиреоидной пероксидазе (АТ-ТПО) в сыворотке крови определяли методом твердофазного иммуноферментного анализа с использованием наборов реагентов «Алкор - Био», (Россия). Результаты рассчитывали на микро планшетном ридере «Stat - Fax - 2100», Awareness Technology Inc, (США). Для системной диагностической оценки функционального состояния гипофизарно - тиреоидной системы рассчитывали вспомогательные индексы: индекс периферической конверсии - показатель периферического дейодирования (ИПК = об. Т4/об. Т3), отношение (с. Т4/ТТГ), отношение (с. Т3/ТТГ).

\section{Статистический анализ}

Рассчитывались средние величины, их стандартные средние ошибки $(\mathrm{M} \pm \mathrm{m})$, а также 95\% доверительный интервал. Для сравнения независимых 
групп использовали $\mathrm{t}$ - критерий Стьюдента. Проверка гипотез о равенстве средних значений проводилась с помощью парного критерия Вилкоксона.

Анализ корреляционных связей был проведен стандартным методом математического моделирования с помощью программы «Statistica Advansed» v. 10.01 StatSoft, (США) в модулях «корреляция», «регрессия», «множественная регрессия». Уравнения множественной регрессии рассчитывали по методу Крамера. Значимость факторов уравнений регрессии оценивали по $\mathrm{F}$ - критерию Фишера. Парные корреляции Пирсона исследовались между всеми показателями для исключения автокорреляций. Результаты обработаны на ПК и считались статистически значимыми при коэффициенте достоверности $\mathrm{p}<0,05$.

\section{Результаты}

Уровни средне динамического артериального давления (АДСр, мм.рт.ст.) в

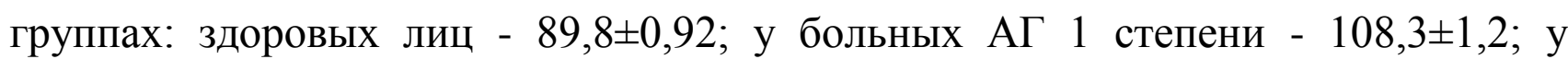
больных АГ 2 степени - 112,6 $\pm 1,8$.

Концентрация стабильных метаболитов оксида азота - NO - нитритов NO2- и нитратов - NO3- и их суммарного содержания в крови - NOx, в группах больных АГ 1 и АГ 2 степени и в группе здоровых лиц представлены в таблице 1.

Таблица 1 Концентрация стабильных метаболитов оксида азота (NO) - нитритов и нитратов в крови у здоровых лии и 8

\begin{tabular}{|c|c|c|c|}
\hline Группы & $\begin{array}{c}\text { Здоровые } \\
\text { лица } \\
(\mathrm{n}=70)\end{array}$ & $\begin{array}{c}\text { Больные } \\
\text { АГ 1 степени } \\
(\mathrm{n}=55)\end{array}$ & $\begin{array}{c}\text { БГ } 2 \text { степени } \\
(\mathrm{n}=60)\end{array}$ \\
\hline Значения & $24,2 \pm 1,4$ & $21,6 \pm 1,3$ & $16,2 \pm 1,4 * \#$ \\
\hline $\mathrm{NO}_{2}^{-}$в крови, мкмоль/л & $114,7 \pm 2,6$ & $107,9 \pm 3,2$ & $83,4 \pm 2,5 * \#$ \\
\hline $\mathrm{NO}_{3}{ }^{-~ в ~ к р о в и, ~ м к м о л ь / л ~}$ & $138,9 \pm 2,3$ & $129,5 \pm 2,6$ & $99,6 \pm 2,2 * \#$ \\
\hline NОх в крови, мкмоль/л & & &
\end{tabular}

Примечания: NO2- - нитриты, NO3- - нитраты, NOx - суммарная концентрация нитритов и нитратов в крови (мкмоль/л.), * - различия достоверны между группами больных АГ и группой здоровых лиц, $\mathrm{p}<0,05$,

\# - различия достоверны между группами больных АГ 1 и АГ 2 степени, $\mathrm{p}<0,05$.

Как видно из таблицы 1, концентрация нитритов - NO-2 в крови в группе больных АГ 1 степени снижена (на 12\%) и достоверно ниже в группе больных АГ 2 (на 49\%), чем у здоровых жителей высокогорья. Концентрация нитратов NO-3 незначительно снижена в крови у больных АГ 1 степени (на 6\%) и достоверно снижена в группе больных АГ 2 степени (на 37\%). Суммарная 
концентрация нитритов и нитратов в крови - NOx у больных АГ 1 степени снижена (на 7\%) и достоверно ниже в группе больных АГ 2 степени (на 39\%), чем в группе здоровых лиц.

Из таблицы видно, что с увеличением степени АГ суммарная концентрация стабильных метаболитов NO - нитратов и нитритов, прогрессивно снижается.

Концентрация гипофизарных и тиреоидных гормонов, антитела к тирео пероксидазе, гипофизарно - тиреоидные индексы представлены в таблице 2.

Таблица 2

Концентращия тиреотропного гормона, тиреоидных гормонов в крови, гипофизарно- тиреодные индексы у здоровых лиц и в группах больных АГ 1 и АГ 2 степени. $(M \pm m)$

\begin{tabular}{|c|c|c|c|}
\hline Группы & $\begin{array}{c}\text { Здоровые } \\
\text { лица } \\
(\mathrm{n}=70)\end{array}$ & $\begin{array}{c}\text { Больные } \\
\text { АГ 1 степени } \\
(\mathrm{n}=55)\end{array}$ & $\begin{array}{c}\text { Больные } \\
\text { АГ 2 степени } \\
(\mathrm{n}=60)\end{array}$ \\
\hline ТТГ, мкМЕ/л & $1,08 \pm 0,01$ & $1,04 \pm 0,02$ & $0,92 \pm 0,02 *$ \\
\hline об. Т3, пмоль/л & $0,71 \pm 0,02$ & $0,68 \pm 0,01$ & $0,59 \pm 0,02 *$ \\
\hline с. Т3, пмоль/л & $1,93 \pm 0,02$ & $1,89 \pm 0,03$ & $1,73 \pm 0,02 *$ \\
\hline об. Т4, пмоль/л & $52,3 \pm 1,2$ & $49,9 \pm 1,1$ & $45,3 \pm 1,3 *$ \\
\hline с. Т4, пмоль/л & $9,2 \pm 0,3$ & $8,4 \pm 0,2$ & $6,8 \pm 0,3 *$ \\
\hline об. Т3/ТТГ, ед. & $0,66 \pm 0,02$ & $0,65 \pm 0,01$ & $0,64 \pm 0,02$ \\
\hline с. Т4/ТТГ, ед. & $8,52 \pm 0,01$ & $8,07 \pm 0,02$ & $7,39 \pm 0,03 *$ \\
\hline об.Т4/об.Т3, ед. & $73,6 \pm 1,3$ & $73,4 \pm 1,1$ & $76,7 \pm 1,2$ \\
\hline АТ-ТПО, мкМЕ/мл & $5,22 \pm 0,11$ & $4,92 \pm 0,1$ & $4,68 \pm 0,12^{*}$ \\
\hline
\end{tabular}

Примечания: (ТTГ, мкМЕ/л), тиреотропньй гормон; общий трийодтиронин (об. Т3, пмоль/л); свободньй трийодтиронин (св. Т3, пмоль/л); общий тироксин (об. Т4, пмоль/л); свободный тироксин (св.Т4, пмоль/л); отношение об. ТЗ к ТТГ (об. ТЗ/ТТГ, ед.); отношение общего тироксина к ТТГ (об. Т4/ТTГ, ед.); отношение свободного тироксина к ТТГ (св. Т4/ТTГ, ед.); индекс периферической конверсии (T4/T3, ед.); антитела к тиреопероксидазе (АТ-ТПО, мкМЕ/мл).

Как видно из таблицы 2, концентрация тиреотропного гормона - ТТГ снижена в группе больных АГ 1 степени и достоверно снижена в группе больных АГ 2 степени по сравнению с группой здоровых лиц. Концентрация общего Т3 снижена в группах больных АГ 1 и АГ 2 степени. Концентрация свободного тироксина (с. Т4) в группах больных АГ 1 и АГ 2 степени снижена по сравнению с группой здоровых лиц. Отношение об. Т3 к ТТГ незначительно снижено в группе больных АГ 2 степени. Отношение с. Т4 к ТТГ снижено у АГ 2 степени. Индекс периферической конверсии (Т4/T3) увеличен в группе больных АГ 2 степени. Антитела к тиреопероксидазе (АТ-ТПО) достоверно снижены в группах больных АГ 2 степени.

Полученные данные свидетельствуют о гипофункции щитовидной железы у здоровых лиц, более выраженную у больных АГ 2 степени. 
Известно, что большая часть тиреоидных гормонов образуются в тканях и около $20 \%$ синтезируется и выделяется щитовидной железой. Адекватное обеспеченье ТГ не возможно при низком тканевом кровоснабжении. Учитывая роль оксида азота в кровообращении и вазодилатации, можно констатировать его важную роль в адекватном обеспечении тиреоидного гомеостаза организма[10].

Дисфункция эндотелия (ДЭ) со снижением продукции NO на фоне снижения концентрации ТГ, вносит существенный вклад в регуляцию артериального давления (АД) и распределения крови в сосудистой сети. Снижение концентрации NO в крови вызывает дисбаланс многих других эндотелий - зависимых факторов: коагуляции, агрегации клеток крови, цитоструктуры сосудов. NO влияет на ростовые факторы, пролиферацию и апоптоз кадиомиоцитов, регулирует сократительную функцию миокарда, усиливает релаксацию желудочков и влияет на многие другие факторы, определяющие течение и прогноз больных с АГ [10,11].

Существенную роль в реализации многих процессов, опосредованных NO, играют ТГ как на уровне клеточных мембран, так и на уровне ядерных структур. При этом эффекты NO и ТГ могут, как потенцировать, так и снижать взаимовлияние на разные физиологические и патологические процессы в органах и тканях [12].

У больных АГ 1 степени на фоне незначительного снижения содержания стабильных метаболитов NO в крови, снижена концентрация ТТГ, общего и свободного Т3, общего и свободного Т4, ниже отношение с. Т4/ТTГ, концентрация АТ - ТПО в крови. Параметры центральной гемодинамики незначительно увеличены. ДЭ у больных АГ 1 степени не приводит к существенным гемодинамическим и биохимическим сдвигам. При наличии гипотиреоза у больных АГ, жителей высокогорья не высокие уровни АДСр.

Более типичные для АГ клинические и биохимические сдвиги наблюдаются в группе больных АГ 2 степени. У них на фоне высоких уровней системного АД выражена дисфункция эндотелия, проявляющаяся в значительном снижении концентрации стабильных метаболитов NO по сравнению с группой здоровых лиц.

ДЭ у больных АГ 2 степени сочетается с нарушениями гемодинамики, высоким уровнем АДСр и более выраженной гипофункцией щитовидной железы. У них снижен общий Т3, свободный тироксин Т4, концентрация ТТГ, 
отношение св. Т4/ТТГ, антитела к тиреопероксидазе (АТ - ТПО). Эти данные свидетельствуют о развитии гипотиреоза в группе больных АГ 2 степени.

Таким образом, значительное снижение концентрации ТГ и продукции NO в группе больных АГ 2 степени приводит к выраженной ДЭ, которая сопровождается вовлечением множества системных компенсаторных механизмов регуляции для ответа на гемодинамические, гормональные и другие стимулы.

Проведен парный и множественный корреляционный анализ и вычислен ряд уравнений множественной регрессии, который показал закономерности отражающие зависимость абсолютных и относительных значений показателей гемодинамики от концентрации NO и тиреоидных гормонов в крови.

При помощи уравнений множественной регрессии в группе здоровых лиц и больных АГ 1 и АГ 2 степени установлены взаимосвязи между концентрацией NO в крови, концентрацией тиреоидных гормонов и уровнем АДСр.

В группе больных АГ 1 степени между концентрацией NO в крови и АДСр, с. Т4, АТ-ТПО установлены обратные взаимосвязи ( $\mathrm{r}=-0,453$ - 0,616; $\mathrm{p}<0,01)$ и прямые слабые взаимосвязи между АДСр и отношением об. Т4/об. Т3 $(\mathrm{r}=0,393 ; \mathrm{p}<0,01)$, слабовыраженные или отсутствующие в группе здоровых лиц.

В группе больных АГ 2 степени между концентрацией NO в крови и АДСр, с. Т3, с. Т4, АТ-ТПО установлены обратные взаимосвязи (r=-0,438 0,493; р<0,01). Прямые взаимосвязи установлены между АДСр и с. Т3, с. Т4, $(\mathrm{r}=0,388-0,468 ; \mathrm{p}<0,01)$. Между концентрацией NO в крови и ТТГ выявлена прямая слабая взаимосвязь ( $=0,385 ; \mathrm{p}<0,01)$.

Выявлена обратная взаимосвязь между концентрацией NO в крови, ТТГ и возрастом у больных АГ (r=-0,594; $<<0,001)$ и в группе здоровых лиц (r=-0,408; $\mathrm{p}<0,01)$, а также обратная взаимосвязь между концентрацией NO в крови и длительностью течения АГ (r=-0,542; $\mathrm{p}<0,001)$.

Концентрация NO в крови тесно связана с центральной гемодинамикой, гормонами гипофиза и учувствуют в реализации как благоприятных, так и патологических влияний на функции эндотелия и метаболизм ТГ. Сложность изменения регуляций определяется не только ее выраженностью, но и значительным временным промежутком при которых эти регуляции достигают целевого уровня функционирования, так как взаимосвязи ТГ и NO осуществляются не только на тканевом уровне, но и на субклеточном и ядерном.

В развитии и течении АГ задействована многоуровневая система патологии систем регуляции, взаимозависимых и взаимосвязанных между 
собой, что приводит к развитию изменений в других органах и способствует возникновению характерных для АГ осложнений.

Существенный вклад в увеличение риска осложнений у больных $А Г$, кроме высокого системного АД вносят другие факторы. Анализ уравнений множественной регрессии показал, что у больных АГ риск развития осложнений регуляции центральной гемодинамики зависит от концентрации метаболитов оксида азота и тиреоидных гормонов.

\section{Заключение}

Таким образом, течение АГ у жителей высокогорья сопровождается снижением стабильных метаболитов оксида азота и тиреоидных гормонов в крови, более выраженное у больных АГ 2 степени.

У больных АГ выявлены обратные взаимосвязи между концентрацией NO в крови, уровнем АДСр и концентрацией тиреоидных гормонов.

У здоровых лиц взаимосвязи между концентрацией NO в крови, уровнем АДСр и концентрацией тиреоидных гормонов слабо выражены или отсутствуют.

\section{Литература}

1. Cheng SY, Leonard JL, Davis PJ. Molecular aspects of thyroid hormone actions. Endocr Rev. 2010; 31: 139-170.

2. Mittag J, Lyons DJ, Sallstrom J, et al. Thyroid hormone is required for hypothalamic neurons regulating cardiovascular functions. J Clin Invest. 2013; 23(1): 509-516.

3. Dillmann WH. Cellular action of thyroid hormone on the heart. Thyroid. 2002; 12: 447-452.

4. Jabbar A, Pingitore A, Simon H. S. Pearce, et al. Thyroid hormones and cardiovascular disease. Nature Reviews Cardiology. 2017; 14: 39-55.

5. Fliers E, Klieverik LP, Kalsbeek A. Novel neural pathways for metabolic effects of thyroid hormone. Trends Endocrinol Metab. 2010; 21(4): 230-236.

6. Beall CM, Laskowski D, Erzurum SC. Nitric oxide in adaptation to altitude. Free. Radic Biol Med. 2012; 52: 1123-1134.

7. Umbrello M, Dyson A, Feelisch M. et al, The key role of nitric oxide in hypoxia: hypoxic vasodilatation and energy supply-demand matching. Antioxid Redox Signal. 2013; 19(14):1690-1710.

8. 2013 ESH/ESC Guidelines for the management of arterial hypertension. The Task Force for the management of arterial hypertension of the European Society of Hypertension (ESH) and of the European Society of Cardiology (ESC). J Hypertens. 2013; 31:1281-1357.

9. Tsikas D. Methods of quantitative analysis of the nitric oxide metabolites nitrite and nitrate in human biological liquids. Free Radical Research 2005; 39(8): 797-815.

10. Петрищев, Н.Н. Дисфункция эндотелия. Причины, механизмы, фармакологическая коррекция. СПб.: СПб ГМУ, 2003. - 184 с.

11. Danzi S., Klein I. Thyroid hormone and the cardiovascular system. Minerva Endocrinologica. 2004; 29: 139-150.

12. Danzi S, Klein I. Thyroid hormone and blood pressure regulation. Curr Hypertens Rep. 2003; 5:513-520. 